\title{
System dynamics and cybernetics: \\ a synergetic pair
}

Markus Schwaninger ${ }^{a *}$ and José Pérez Ríos ${ }^{\mathrm{b}}$

Markus Schwaninger is professor of Management at the University of St. Gallen, Switzerland. His research is focussed on Organizational Cybernetics and System Dynamics, applied to issues of organizational intelligence, management systems, and to the design, transformation, and learning of social systems.

José Pérez Ríos is professor of Business Organization in the University of Valladolid, Spain. His research is focussed on the application of System Dynamics and Management Cybernetics to the study of complex systems, and to the development of software tools which can facilitate the application of different systemic approaches as well as knowledge capturing, communication and information exchange.

\begin{abstract}
The authors advocate building a bridge between two systems approaches, namely system dynamics (SD) and the viable system model (VSM), which is the main exponent of organizational cybernetics (OC). Such a synthesis is aimed at opening a path towards a better capability of actors to deal with complex issues in both organizations and society. Given their respective strengthsmodeling and simulation of content issues in the case of SD, and providing a viable organizational context in the case of OC-a combination of the two approaches is claimed to be potentially fertile. That argument gets twofold support. Firstly, the complementarity of SD and the VSM is cogently shown. Secondly, the authors refer to examples of synergy development through a combination of the two approaches. Copyright (c) 2008 John Wiley \& Sons, Ltd.
\end{abstract}

Syst. Dyn. Rev. 24, 145-174, (2008)

\section{Introduction}

In this paper we identify a complementarity between the two methodological disciplines ${ }^{1}$ of system dynamics (SD) and organizational cybernetics (OC) (or managerial cybernetics $)^{2}$ for applications to social systems. We propose that this complementarity should be explored synergistically. We shall also give an outline for building a bridge between SD and OC. One does not build a bridge for the sake of construction, but because one wants to establish a necessary connection.

The purpose of this paper is to show why and how SD and OC are complementary. Both disciplines are rooted in the systems approach, and therewith have bred methodologies for dealing with complexity. Both originate from a common theoretical basis: general system theory and information theory. But each one also has additional roots in disciplines or theories which are specific to it, in particular, neurophysiology and set theory for managerial cybernetics, and engineering and control theory for SD.

SD is based on a strong idea: capturing the underlying characteristics of complex dynamic systems by means of modeling and simulation, in order to understand them better, and to enable the design of policies that:

- foster desirable developments; as well as

- make errors along the way less likely.

\footnotetext{
${ }^{a}$ Institute of Management, University of St Gallen, Dufourstr. 40a, St Gallen CH-9008, Switzerland.

${ }^{\mathrm{b}}$ Departamento de Organización de Empresas, Universidad de Valladolid, Valladolid, Spain.

* Correspondence to: Markus Schwaninger. E-mail: markus.schwaninger@unisg.ch

Received January 2005; Accepted April 2008
}

System Dynamics Review Vol. 24, No. 2, (Summer 2008): 145-174

Published online in Wiley InterScience

(www.interscience.wiley.com) DOI: 10.1002/sdr.400

Copyright ( 2008 John Wiley \& Sons, Ltd. 
The SD methodology, ${ }^{3}$ in combination with the development of excellent software packages, has strengthened the momentum of both SD-based practical applications and model-based theory building in general.

SD has become a powerful approach to dealing with dynamic complexity. Even so, it is not a panacea, for it often needs completion from without. Depending on the issues under study, system dynamicists are well advised to include complementary methodologies in their toolkit.

Lane and Oliva (1998) have highlighted one such complementarity. These authors ascertained a lack within SD of theories for generating and representing diverse issues and for enhancing sensitivity to socio-political aspects. Therefore they proposed a synthesis between SD and the soft systems methodology (SSM) of Checkland (1981).

We agree with this proposal. It leads to complementing logic-based analysis of the issues at hand with an "extended cultural analysis" which incorporates analyses of the intervention as well as the social and political systems (Lane and Oliva, 1998, p. 228). The present paper continues in that vein, with a focus on organizational development and problem solving.

When dealing with complex issues or problems in organizations, content, context and process should be studied and designed simultaneously, as postulated by the organizational scientist Andrew Pettigrew (1985, p. 50). ${ }^{4}$ To clarify these three terms, we are using the following definitions. Content is that which is contained in a system or, more specifically, in an issue at hand (after Oxford English Dictionary, 1996, p. 324/815). By context we understand the (larger) connection or environment into which a content is woven (Oxford English Dictionary, 1996, p. 325/820f.). Finally, process is defined as a series of actions or events (Oxford English Dictionary, 1996, p. 1436/545) which manifest or are linked to the dynamics of the system under study. In the case of problem solving, content signifies what the problem is about, which are its components and how these interact. Context is the organizational environment in which the content is embedded. Process is the sequence of activities by which the problem solving manifests itself, and in particular the set of interventions by which it is managed.

The rationale of our argument in a nutshell is as follows: SD provides a methodology for modeling and simulating organizational issues dynamically. A large spectrum of issues of strategy or operations can be represented, and pertinent decision making at the content level can be supported effectively. However, for the analysis and design of the organizational context complementary models are needed (see Espejo, 1993). Such models can be provided by cybernetics.

Specifically, we propose the combination of SD and the viable system model (VSM; see Beer, 1979, 1981, 1985), which is the best-known model of OC. This combination is advocated in view of a distinctive characteristic of that model: it is not only a blueprint for modeling organizations as viable systems. The VSM also specifies-as it claims-the necessary and 
sufficient structural preconditions for the viability of any organization, which makes it a unique, very powerful device for the diagnosis and design of organizations. Despite its strong theoretical claim, the model has not been refuted so far. ${ }^{5}$ As we shall see, SD and the VSM can complement each other synergistically. When addressing the VSM, we will mainly refer to the methodology of organizational diagnosis and design, linked to the model.

Like SD, the VSM is powerful but limited in the issues it can deal with. Namely, it lacks specific features for capturing the dynamic aspects of complex systems. That is why it needs to be complemented.

The issues of methodological complementarities, particularly with regard to SD and the VSM, have been hardly explored in the literature. Among the exceptions are Jackson (2000, pp. 364-367), Mingers and Gill (1997) and Schwaninger (2004).

In order to understand the complementarities of SD and the VSM better, we will be setting forth analyses of both methodologies with a view to their respective strengths and limitations. This will then lead us to their complementarities and to our proposal for integrating or combining the two. Finally, we are going to reflect on the further research needed in order to accomplish such an integration of SD and OC.

We have avoided burdening our exposition with detailed elaborations of questions of process, e.g., the sequencing of events, the modes of observing activities and the methodology of interventions. The process issues, which have been treated elsewhere, ${ }^{6}$ are therefore not addressed specifically in this paper.

\section{System dynamics: strengths and limitations}

The strengths and limitations of the SD methodology are a consequence of its specific characteristics. In the context of the multiple theories and methodologies of the systems movement, the distinctive features of SD are: ${ }^{7}$

- Feedback as conceptual basis. The SD model systems are high-order, multiple-loop networks of closed loops of information. Concomitantly, an interest in nonlinearities, long-term patterns and internal structure rather than external disturbances is characteristic of SD (Meadows, 1980, p. 31). However, SD models are not "closed systems", as sometimes is claimed, in the sense that (a) flows can originate from outside the system's boundaries, (b) representations of exogenous factors or systems can be incorporated into any model as parameters or special modules, and (c) new information can be accommodated via changes to a model. In other words, the SD view hinges on a view of systems, which are closed in a causal sense, but not materially (Richardson, 1991, p. 297). 
- Focus on internally generated dynamics. SD models are conceived as closed systems. The interest of users is in the dynamics generated inside those systems. Given the closed nature of feedback loops and the fact that delays occur within them, the dynamic behavior of these systems is essentially nonlinear.

- Emphasis on understanding. For system dynamicists the understanding of the dynamics of a system is the first goal to be achieved by means of modeling and simulation. Conceptually, they try to understand events as embedded in patterns of behavior, which in turn are generated by underlying structures. Such understanding is enabled as SD "shows how present policies lead to future consequences" (Forrester, 1971, p. VIII). Thereby, the feedback loops are "a major source of puzzling behavior and policy difficulties" (Richardson, 1991, p. 300). SD models purport to test mental models, hone intuition and improve learning (cf. Sterman, 1994, p. 119f.).

- High degree of operationality. SD relies on formal modeling. This fosters disciplined thinking; assumptions, underlying equations and quantifications must be clarified. Feedback loops and delays are visualized and formalized; therewith the causal logic inherent in a model is made more transparent and discussable than in most other methodologies (Richmond, 1997). Also, a high level of realism in the models can be achieved. SD is therefore apt to support decision-making processes effectively.

- Far-reaching requirements (and possibilities) for the combination of qualitative and quantitative aspects of modeling and simulation. This is a consequence of the emphasis on understanding. The focus is not on point-precise prediction, but on the generation of insights into the patterns generated by the systems under study (Meadows et al., 1982).

- High level of generality and scale robustness. The representation of dynamic systems in terms of stocks and flows is a generic form, which is adequate for a wide spectrum of potential applications. This spectrum is both broad as to the potential subjects under study, ${ }^{8}$ and deep as to the possible degrees of resolution and detail (La Roche and Simon, 2000). In addition, the SD methodology enables one to deal with large numbers of variables within multiple interacting feedback loops (Forrester, 1969, p. 9).

- Availability of powerful application software. The packages (Stella/Ithink, Powersim, VENSIM and MyStrategy) ${ }^{9}$ are easy to handle and give access to a high variety of mathematical functions. Some of them offer optimization procedures and validation tools (cf. Eberlein and Peterson, 1994). Other useful features include support for collaborative modeling and communication with databases. These features related to SD are not unique in an absolute sense, but they are distinctive in comparison with the software available for the support of VSM-based diagnosis and design.

The features of SD just sketched out result in both strengths and limitations. We start with the strengths. 


\section{Strengths of SD}

1. Its specific modeling approach makes SD particularly helpful in gaining insights into the patterns exhibited by dynamical systems, as well as the structures underlying them. Closed-loop modeling has been found most useful in fostering understanding of the dynamic functioning of complex systems. Such understanding is especially facilitated by the principle of modeling the systems or issues under study in the continuous mode and at rather high aggregation levels (Forrester, 1961; La Roche and Simon, 2000). With the help of relatively small but insightful models, and by means of sensitivity analyses as well as optimization heuristics incorporated in the application software packages, decision spaces can be thoroughly explored. Vulnerabilities and the consequences of different system designs can be examined with relative ease.

2. The generality of the methodology, and its power to crystallize operational thinking in realistic models, have triggered applications in the most varied contexts. Easy-to-use software and the features of screen-driven modeling via graphic user interfaces provide a strong lever for collaborative model building in teams (cf. Vennix, 1996; Andersen and Richardson, 1997).

3. Another strong point is the momentum of the SD movement. Owing to the strengths commented on up to this point, the community of users has grown steadily, being probably the largest community within the systems movement. ${ }^{10}$ The use of SD has transcended disciplinary boundaries, ranging from the formal and natural sciences to humanities, and covering multiple uses from theory building to education and to the tackling of real-world problems at almost any conceivable level. Applications to organizational, societal and ecological issues have seen a particularly strong growth. This feeds back on the availability and growth of the knowledge the individual modeler can draw upon.

4. Its specific features make SD an exceptionally effective tool for conveying systemic thinking to anybody. Therefore, it also has an outstanding track record of classroom applications for which "learner-centered learning" is advocated (Forrester, 1993, 1997). The pertinent audiences range from schoolchildren at the levels of secondary and primary schools to managers and scientists.

The flipside of most of these strengths embodies the limitations of SD; we concentrate on those which can be relevant to a possible complementarity of SD with the VSM.

\section{Limitations of $S D$}

1. The main point here is that SD does not provide a framework or methodology for the design of organizational structures conceived as patterns of 
relations among organizational actors, including the distribution of activities and functions. ${ }^{11}$ This other concept of structure, which comes from cybernetics, is distinct from the concept used in SD, which is about causal structures of problems represented by means of stocks, flows and feedback loops. The levels of analysis in SD, then, are patterns of behavior embracing the events and structures that underlie those patterns.

The lack of a framework for the design of organizational structure makes SD susceptible to completion from without-a completion which OC, and the VSM in particular, could provide (cf. Schwaninger et al., 2004). The choice falls on the VSM because of its strong heuristic power and its complementary strengths in relation to SD. This will become clear in the next section, where the VSM will be expounded.

2. Another limitation of SD is related to the absorption of variety ${ }^{12}$ (complexity) by an organization. SD offers an approach to the handling of variety which allows modeling at different scales of a problem or system (Odum and Odum, 2000). It focuses on the identification, at a certain resolution level or possibly several resolution levels, of the main stock variables which will be affected by the respective flows. These in turn will be influenced by parameters and auxiliary variables. This approach, even though it enables thinking and modeling at different scales, does not provide a formal procedure for an organization to cope with the external complexity it faces, namely for designing a structure apt to absorb that complexity. In contrast, OC offers an elaborate model to enable the absorption of variety (complexity), based on Ashby's law of requisite variety ${ }^{13}$-the VSM. The VSM has two salient features in this respect. Firstly, it helps design an organizational unit in a way that enables it to attenuate the complexity of its environment, and also to enhance its eigen-variety, so that the two are in balance. ${ }^{14}$ Secondly, the recursive structure of the VSM ensures that an organization with several levels will develop sufficient eigen-variety along the fronts on which the complexity it faces unfolds. These two features can deliver a strong complement to SD.

It follows that there is a need to complement SD with other methodologies, when issues are at stake, which it cannot handle by itself. We maintain that the VSM is an excellent choice, when issues of organizational diagnosis or design are to be tackled. At the same time, SD can be a powerful complement to other methodologies-to the VSM in particular. The perspective on these aspects will be deepened in the following section.

\section{The viable system model: strengths and limitations}

The cybernetic view of socio-technical systems has bred models and methods for management in general and for the diagnosis and design of organizations in 
particular. Laying out the whole theory of OC would transcend the scope of this paper. We shall focus on the viable system model coined by Stafford Beer, the father of OC (Beer, 1979, 1981, 1985, 1989), which is the most wide-ranging organizational theory brought forth to date by that discipline.

Among the distinctive features of the VSM are: ${ }^{15}$

- Focus on viability. The VSM is a framework for the structuring of organizations as viable systems, which deal with complexity adaptively and recursively. This focus on viability does not preclude other theories of management, but it does complement the many approaches which focus on partial aspects of design and control with an integrative view. It also provides a theory for the design of organizations and parts thereof as wholes, the viability and development of which are grounded in their ability to cope with complexity effectively.

- High level of generality. The VSM is not concerned with a particular structure, but with a system's essential organization-with what defines the system and enables the maintenance of its identity (Jackson, 1988, p. 560). In a nutshell, the VSM specifies a set of functions, which provide the necessary and sufficient conditions for the viability of any human or social system. These functions and their interrelationships are specified in a comprehensive theory (Beer, 1979, 1981, 1985), which is, in principle, applicable to any social system, particularly to organizations (see below).

- Theoretical propositions. ${ }^{16}$ The main theoretical proposition stipulated by the VSM is that an organization is viable if and only if it has a set of management functions and their interrelationships as specified by the theory. To our knowledge, this proposition is stronger than those of any other theory of organization design. The second proposition is that any deficiencies in this system, such as missing functions, insufficient capacity of the functions or communication channels, or faulty interaction between the functions, impair or endanger the viability of the organization. The third proposition is that the viability, cohesion and self-organization of an enterprise depend upon these functions being recursively operative at all levels of the organization. A recursive structure comprises autonomous units within other autonomous units. Moreover, a viable organization is made up of viable units and itself forms a part of other, more comprehensive viable units.

The set of the management functions and their interrelationships identified and formalized in the VSM are as follows (Figure 1). The management functions are represented by the boxes named " 1 " to " 5 " and refer to Systems 1 to 5 :

- System 1: Regulatory capacity of the basic units (A, B, C, D); autonomous adaptation to their environment; optimization of ongoing business (e.g., the business areas of a company). 
Fig. 1. Stafford Beer's viable system model (overview) $)^{17}$

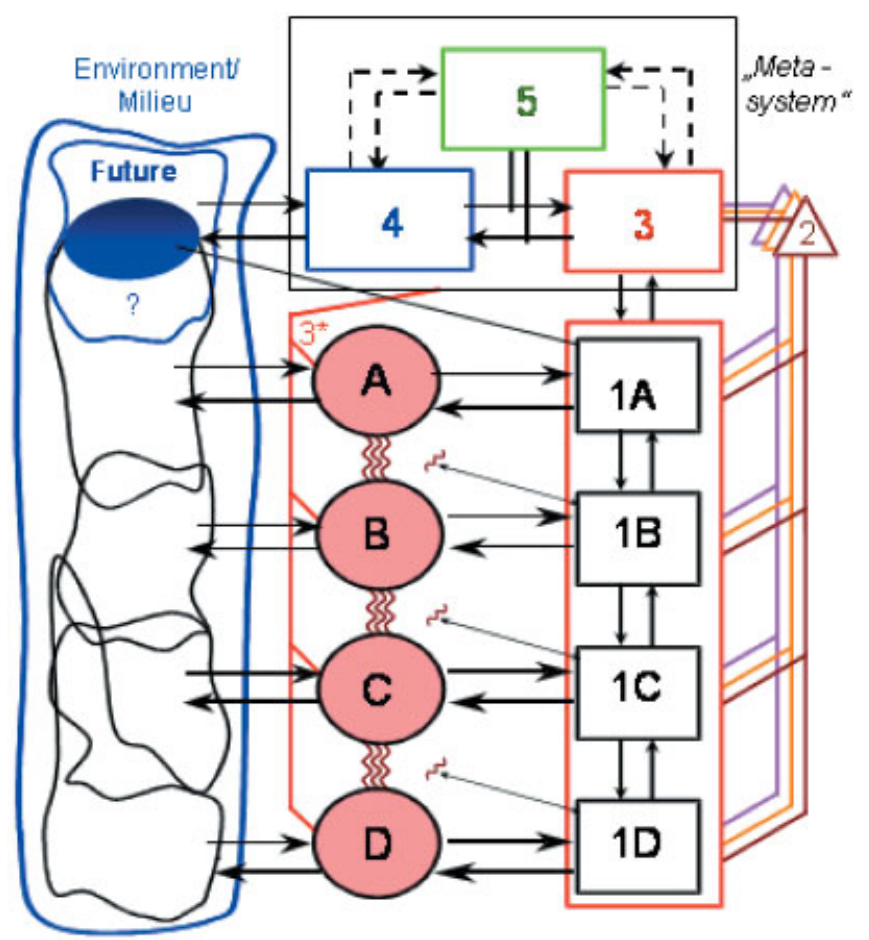

- System 2: Amplification of self-regulatory capacity; attenuation of oscillations and coordination of activities via information and communication (e.g., information systems, service units and coordination teams, standards of behavior, knowledge bases).

- System 3: Management of the collective of primary units (basic units with regulatory capacity); establishment of an overall optimum among basic units, provision for synergies as well as resource allocation (e.g., the executive corporate management).

- System 3*: Investigation and validation of information flowing between Systems $1-3$ and 1-2-3 via auditing/monitoring activities (e.g., operations analysts, special studies and surveys).

- System 4: Management of the development of the organization; dealing with the future-especially the long term-and with the overall outside environment; diagnosis and modeling of the organization in its environment (e.g., corporate development, strategy, research and knowledge creation).

- System 5: Balancing present and future as well as internal and external perspectives; moderation of the interaction between Systems 3 and 4; ascertaining the identity of the organization and its role in its environment; embodiment of supreme values, norms and rules-the ethos of the system (normative management). 
In this structure, the primary units (basic units with the regulatory capacity supplied by System 1) must be endowed with high autonomy in order to be able to adapt to their respective environment or milieu. The combined activities of Systems 1, 2 and 3 (including $3^{*}$ ) provide for management of the present and short term, while System 4 is the fulcrum for long-term adaptation, and System 5 is the embodiment of the ethos-the basic principles governing the orientation of the organization as a whole.

Systems 1-2-3 (including $3 *$ ) comprise the operative management, System 4 in interaction with System 3 the strategic management, and System 5 the normative level of management. System 3 is the linchpin between the operational and the strategic levels.

A fundamental idea inherent in the theory of the VSM is that social systems are structured recursively. The meaning of this idea is that the structure described heretofore is applicable to all organizational levels of a system. For example, it applies at the same time to a company, its divisions, the business units which constitute the divisions, etc. Hence, in the ideal case, a viable system is embedded within another viable system and is itself constituted by viable systems (Beer, 1979, 1985).

\section{Strengths of the VSM}

The strengths of the VSM are based on its very strong theoretical claim. The consequence is that the VSM is primarily effective as a device for diagnosing organizations. Beyond that it has proven to be a most helpful heuristic for organizational design, namely under the aspect of the management of variety and the encouragement of self-organization (Jackson, 1988, p. 561). It helps the design of structures when issues of differentiation and integration are at stake. It also supports the proper arrangement of control and information systems (Jackson, 1988, p. 570). Finally, the object of VSM analysis, in addition to structural aspects, which are its main concern, also includes the cultural dimension (see description of System 5 above), which reinforces the points just made.

In conclusion, the generality of the VSM makes it a powerful conceptual tool for both organizational diagnosis and design, independent of the specific features of the organizations under study, such as type of activity, size, location, products, markets, technology and the like. Also, according to a comparison of theories of viability carried out recently, the concision and stringency of the theory, as well as its elaborateness and transparency, are considerable (Schwaninger, 2006a). The fact that the model has not been falsified to date (see above) gives strong support to the assumption that its bold theoretical claim is justified.

These strengths have led to an increasing interest in the VSM. As public response indicates, it is obviously attractive and exerts great fascination, at least as far as the community of academics and practitioners interested in the 
systems approach is concerned (Schwaninger, 2006a). This is shown by the fact that more and more people and organizations now work with it. The VSM is chiefly utilized in general management and consultancy. This applies to both the private and public sectors. Examples are to be found in companywide organization designs, and in diagnoses of the total organization of firms of all kinds and sizes as well as public and international authorities. The VSM has also been employed to analyze the political systems of whole nations, and VSM-based theses on themes related to engineering have been completed at technical universities or institutes in different countries (for details, see Schwaninger, 2006a, and the literature quoted therein).

However, the VSM has not yet become as popular as SD. This is in part due to its later appearance and the fact that its originator never operated from a stable academic base, as opposed to the case of Jay Forrester, the father of SD. In part, too, the slower uptake for VSM may be due to its limitations, which, however, are not altogether limitations intrinsic to the model itself.

Just what are the limitations of the VSM? Several restrictions arise which are related to the model's capabilities as well as to its application.

\section{Limitations of the VSM}

1. As far as its capabilities are concerned, the VSM does not lend any help to the detailed design of organizational infrastructures, e.g., qua division of labor, human resources, detailed information and communication structures. What it does provide is a guideline for the diagnosis of the viability and functional design of the essential organization of a system, which enhances its viability, i.e. makes it capable of controlling itself and maintaining its identity. The model does not give any recommendations with reference to the detailed materialization of a particular structure (Jackson, 2000, p. 172). Most important, the model is not able to deal with the content of organizational activity: How the variables of the system interact, which policies are likely to lead to a certain desired outcome, how particular issues should be solved, etc. The VSM is situated at a higher level of abstraction than most theories about the structuring of organizations. Therefore it is essentially unapt to deal with the content of issues to be handled. It is, however, appropriate for modeling the organizational, namely the structural, context within which these issues are to be coped with (cf. Schwaninger, 2004).

2. The strength of the model as a framework for organizational diagnosis and design in terms of viability is countervailed by another shortcoming. While the VSM does allow one to figure out both the current and the desired states, it does not provide support on how to get from the former to the latter.

Given this limitation, there is a need for additional theories about the realization of the desired functional state, i.e., how it can be realized in terms of, for instance, the way people interact, what their jobs and responsibilities 
are, what information they need, how they should be motivated, etc.; in short, a design theory at the level of the organizational infrastructure. Theories of this kind are highly operational. In addition, they refer to dynamic issues. They are precisely the kind of theory that can be built with the help of SD. SD can make a strong contribution to analysis and design in terms of the dynamics of a system.

3. In terms of applications of the VSM, practitioners have claimed that it is not an easy-to-use model, given the complexity of its theoretical underpinnings (cf. Walker, 2001). Related to this is the lack of specific tools supporting applications of the VSM. Instruments for the simulation and analysis of the dynamic behavior of an organization and its environment, which would enable the exploration of scenarios and assessment of the impact of changes taking place or decisions made, have yet to be provided. SD-based methods and tools would be a welcome complement that would let VSM overcome this drawback.

Just as the VSM would compensate some of the major limitations of SD, the same seems to apply reciprocally.

The main point here becomes palpable if we revert to the distinction between content and context, as introduced above under the first limitation of the VSM. The complementarity of SD and the VSM can be anchored in this distinction. SD is a highly effective device for dealing with the contents of organizational activity, i.e., what the organization aims for, what it does, and where it is heading. That is to say, by means of an SD model one can develop dynamic theories for the implementation of change, and put them to the test for the specific case at hand. In addition, SD models can help to recognize conflict potentials and trade-offs before the change process has even started. They also enable one to design policies that take those into account and thereby minimize the unintended consequences of the required change. All of these features are essential for decision support. They cannot be provided by the VSM, but in many VSM-based studies they would be needed.

The VSM, on the other hand, provides a powerful heuristic for a model-based diagnosis and design of the structural context in which the organizational activities can be carried out, especially for decision processes. It enables one to shape an organization so as to develop these processes in effective ways. If sound decisions about substantive issues can be made on the basis of rational analysis, facilitated by an SD model, VSM-based analysis and design can show where in the structure these decisions should be located. Furthermore, the organizational context can be shaped so as to provide the structural requirements necessary for making the decision happen and getting it implemented. In this sense SD and the VSM are a potentially synergistic pair.

With the advantages and shortcomings of both SD and managerial cybernetics laid out, we can now proceed to the main part, where we develop a more concrete proposal for a synergistic combination of the two approaches. 


\section{The complementarity of SD and the VSM}

In the previous paragraphs we have discussed the strengths and limitations of both SD and VSM. Our analysis has shown that frequently what is a limitation in one approach is a strength in the other, at least at the actual stage of development of both.

Summing up, we have seen that SD is a powerful methodology, which enables the understanding and management of dynamic systems. Nevertheless, SD does not provide a theory for the structural design of organizations. That, however, is precisely one of the greatest strengths of the VSM-it indeed provides a quite complete, coherent and clear theory applicable to the diagnosis and design of any organization.

We have also placed the VSM under scrutiny, and discerned that its major limitation is the difficulty it has in analyzing the dynamic behavior of a complex system. The VSM does provide a conceptual model for depicting the organization in its environment, with an emphasis on management control functions and the interrelationships of control and communication. However, it does not provide any theory about the substantive issues confronted by that organization. In other words, the VSM does not help one to deal with the content of the issues to be tackled, although it helps in modeling the organization so as to make it able to tackle those issues. Instead, it is about structural/ organizational ${ }^{18}$ context, not content.

The dynamic behavior of the organization in its environment is an outcome of the interaction of the system with its environment over time. Modeling and simulating the consequences of different scenarios about the evolution of the environment, studying the impact of decisions, exploring the space of options with their consequences, etc., require a specific modeling and simulation approach which the VSM itself does not provide. The extraordinary capability for meeting these needs is precisely the strength of SD.

Given this complementarity of SD and VSM, we think that their combination is promising. It is high time to braid SD together with cybernetics-which Richardson (1991) described as following a kind of parallel track-in order to build a stronger rope.

In the following paragraphs we will explore in some depth where we think a combination of both methodologies would be most beneficial. First, we will underpin the argument that there exists a need for modeling at both the content and context levels. Next, we will give illustrations of modeling at each of these levels, reverting to examples from a real-life case. Finally, the two threads will be brought together with reference to strategy making.

\section{The need for modeling at both levels: content and context}

Earlier we enumerated the five management functions which are identified in the VSM as necessary and sufficient for an organization to maintain its viability. 
One of these functions is System 4, which is responsible for dealing with the long-term future and the overall outside environment, and the diagnosis and modeling of the organization in its milieu. We need to elaborate on this aspect.

Any system operates in a specific environment, which will change over time. If the system is to keep its viability, it must evolve according to those changes happening in its environment which can have an impact upon it. It is convenient to distinguish between two kinds of environment. The first can be called the accepted environment of an organization (e.g., an enterprise). By that term we refer to what is actually happening in that environment and what the trends are. We discern the second one as a more problematic kind of environment, one particularly related to the unknown future (Beer, 1979).

As Beer specified in his theory, a System 4 is the fulcrum for the adaptation of a viable organization. It embodies the apparatus with which the organization controls itself vis-à-vis the changes happening in its environment and with a focus on the long term. To maintain this crucial regulatory function effectively, the system must contain sufficiently rich models of what it intends to regulate. ${ }^{19}$ In this case, the object of (self-) regulation is the whole viable system, e.g., an organization as it interacts with its environment.

A pertinent model must embody two aspects (cf. Espejo, 1993); Schwaninger, 2004):

(a) the content of these interrelationships, i.e., the organizational activities and issues;

(b) the context of these organizational activities, i.e., the social structure which maintains these interrelationships.

A shorthand description of a System 4 as part of a VSM is contained in Figure 2 (based on Beer, 1979). A System 4 needs comprehensive models of the organization in its environment. In the graph, modeling at both the content and context levels is addressed as the task of System 4. Here, a reference to the complementarity of SD and the VSM is indicated. The need for dynamic images of organizational activities (content level) calls for SD modeling, while the VSM is most helpful in depicting the structure of the organization as a whole (context level). ${ }^{20}$

In sum, we envision the specific contributions of SD and VSM as follows. SD, in a nutshell, provides a model of an issue (or problem) and its evolution over time, and by way of that model it focuses on working out solutions for coping with this issue. The VSM contributes by enabling a diagnosis of the organizational system in which this problem and solution are embedded. In other words, the object dealt with by the VSM occupies a meta-level in relation to the problem treated by the SD model. Each model is useful only at its specific logical level, being of little value if used on the logical level of the other model. 


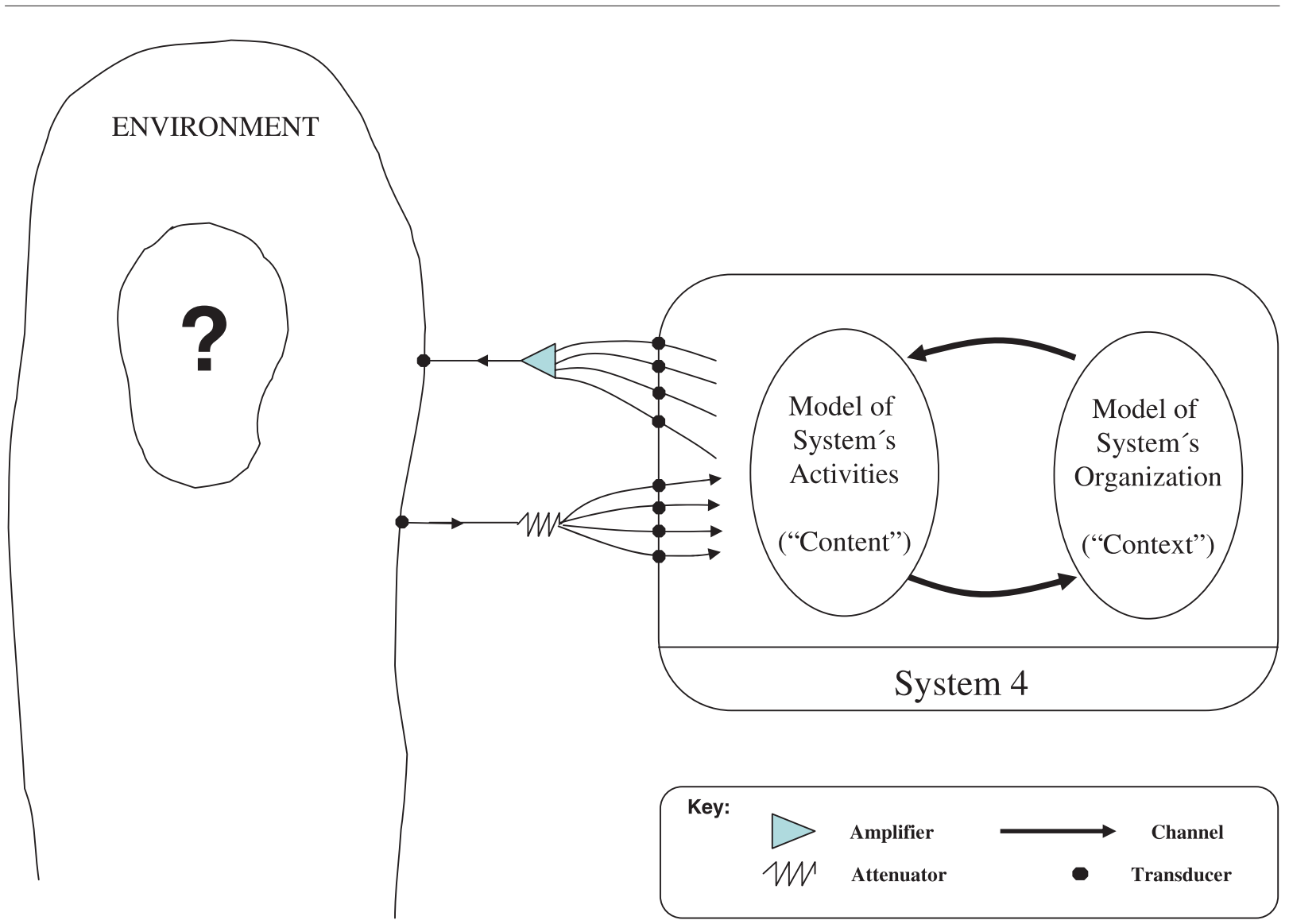

Fig. 2. Two kinds of model in System 4

\section{Modeling at the content level}

Let us first elaborate on the models at the content level. Typical activities related to the System 4 of an enterprise are, for instance, market research, research and development, product design and long-term studies of the organization in its environment. All of these activities handle issues related to the actual and future environment of the enterprise, and, depending on how well they are able to deal with them, its viability will be more or less "safe".

The effectiveness of a System 4 hinges on how good is its model of the organization in its environment. It determines what the management for the long term can achieve or what will be out of reach. Stafford Beer called System 4 activity the management for the "outside and then" (Beer, 1979). As this is a very broad and comprehensive task, the respective models must draw on different sources of knowledge. These sources are multidisciplinary 
Fig. 3. Building a shared understanding with the help of group model building ("interdisciplinary"), but ultimately the integrated view must be holistic, i.e., it must transcend the boundaries of all the disciplines involved. Therefore, the quest is for a shared model, and the modeling task is transdisciplinary; in principle, it transcends the individual sources of knowledge. In addition, management not only needs to fill in content but also to ensure that change in the direction chosen as viable is also feasible. We will first examine the ability of SD for modeling at the content level.

A crucial feature here is the creation of an integrated image through shared understanding of a system by the representatives of distinct views, i.e. a model espoused by the constituents of System 4. Different observers associate diverse contents with a system. They might even conceive the system differently, as far as its boundaries and structures are concerned. In Figure 3, a set of different perspectives is visualized, with some links between them. The SD-based methodology of group model building $(\mathrm{GMB})^{21}$ is then symbolized as an integrative force. Given its transdisciplinary approach, GMB enables an integration of the different perspectives into one shared image of the system-in-focus.

The importance of a shared or aligned understanding is due to the fact that this represents the only way of enabling the adjustments necessary ${ }^{22}$ in order to ensure adaptation and co-evolution with a changing environment.

A practical example is given below. In Figure 4, the process is illustrated with an SD project which supported the development of a Regional Innovation and Technology Transfer System (RITTS) in the region of Aachen, Germany. As reported elsewhere, in a first round a qualitative model of the system under study was established, in which the different views of different stakeholders were integrated (Figure 4, left). ${ }^{23}$ Most of these stakeholders were represented personally by the participants of the two modeling workshops. The result of the qualitative modeling exercise was a shared mental model in the form of a causal loop diagram (Figure 4, right).

This model is about content. It visualizes in essence how the RITTS functions, i.e., how it produces its outcome. It links the interrelationships between operational activities such as product innovation, process innovation, research,

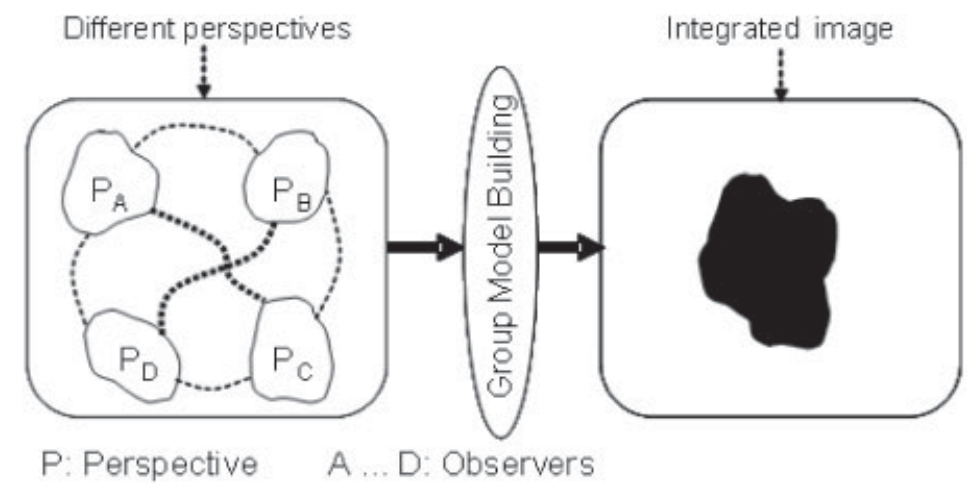

Published online in Wiley InterScience (www.interscience.wiley.com) DOI: 10.1002/sdr 


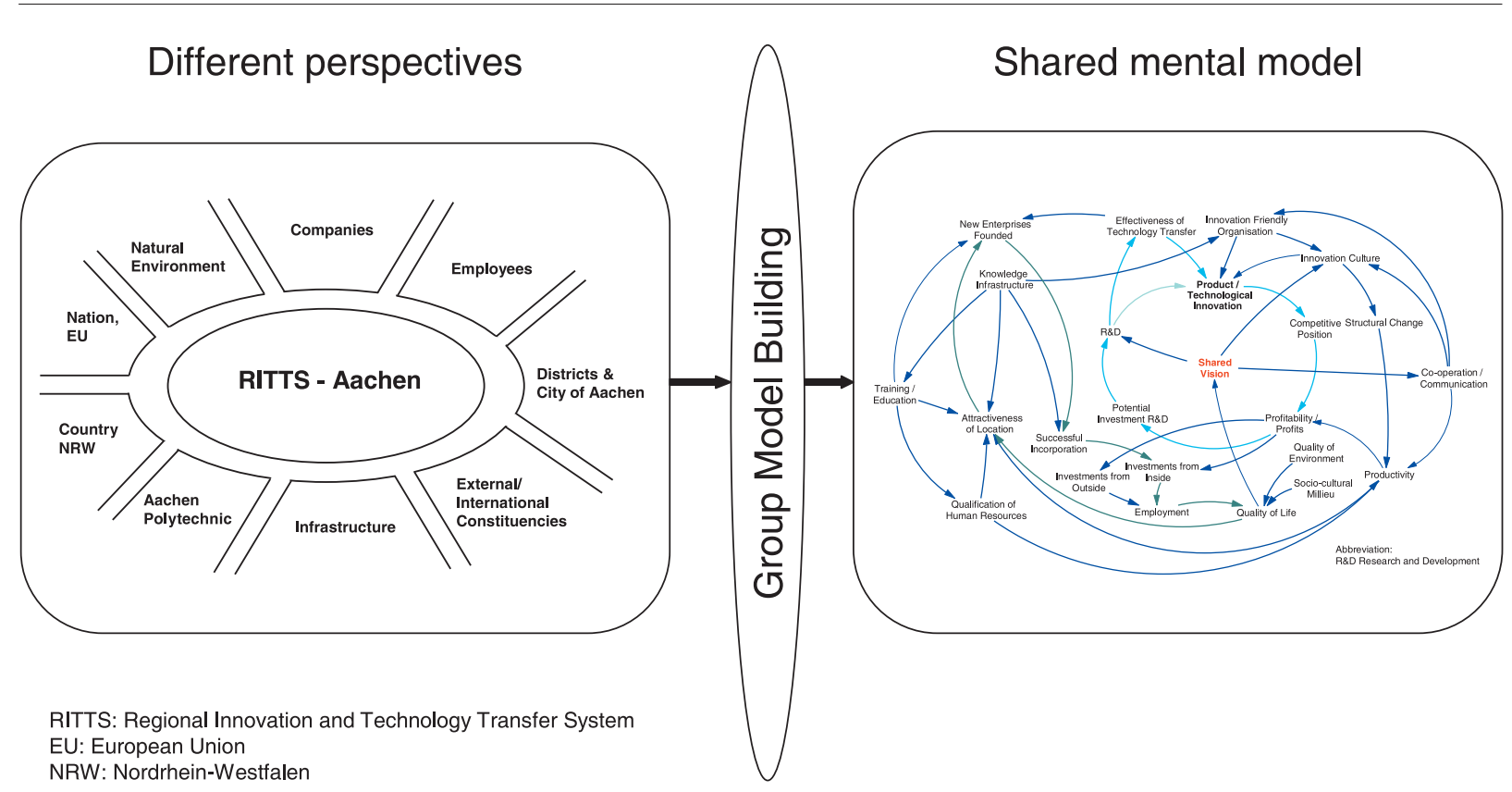

Fig. 4. Formation of a shared mental model (example)

technology transfer, with the strategic prerequisites ("success factors") which make operations and success possible. This kind of qualitative representation only indicates, by the arrows and closed loops, that there are dynamic relationships, but it does not quantify them. Therefore, an even more operational, detailed, quantitative model is often needed. In the case referred to here, such a model was built. The details have been documented elsewhere (Pérez Ríos and Schwaninger, 1996).

Qualitative SD models such as the closed-loop diagrams are good vehicles for producing a first approximation to a complex issue under study in order to reach some preliminary understanding. Beyond that, quantitative models are more useful. This is due to the fact that assumptions can be challenged, tested and then accepted or rejected on the basis of a fairly neutral tool, rather than on the basis of personal preference, position or power within the organization (Sterman, 2000). The same applies to the design and test, by means of simulation, of policies in order to minimize unwanted side effects or unintended consequences.

Concerning the notion of a shared mental model addressed above, a crucial question arises: does shared understanding mean that an organization must have one (and only one) model of the system in its environment? Monistic reliance on one single model is definitely very dangerous: It leads to organizational blindness, with its potentially disastrous consequences. ${ }^{24}$ Hence there is a continuous need to consider, generate and explore a variety of models. It is 
necessary to accept and even foster the coexistence, in the organization, of a diversity of perspectives, opinions and models. Diversity is essential for the survival of an organization. ${ }^{25}$ Agreement in the sense of adherence to one single model may be the result of groupthink (Janis, 1972), and it entails the danger of not seeing important aspects or not considering relevant options. ${ }^{26}$ Consensus also is not a result generally needed for high performance, since it is required in only a limited form: whenever a decision for an action is taken, the actors responsible for it should agree on and stand behind that decision and put it into practice together.

This now brings us to the discussion of the VSM as the appropriate theoretical framework with which to complement SD modeling.

\section{Modeling at the context level}

Having elaborated on models at the content level, we now turn to address modeling at the level of context. We are referring to the social context ${ }^{27}$ within which the organization's activities are carried out. This concerns the structures and processes in which the operations of a social system are embedded. The purpose of modeling at the level of context is to provide a basis for organizational diagnosis and design.

As far as the respective model is concerned, we do not claim that it must necessarily be a cybernetic model. What speaks for the use of the VSM in many cases is that it supplies a very powerful diagnostic tool for telling us how viable an organization is. That utility is, above all, given in cases where an overall diagnosis of the viability or functionality of an organization is required. In addition, the VSM provides an array for supporting the design of organizations, specifically so as to warrant balancing the varieties (complexities) of interacting systems. Another favorable aspect is the versatility of the model. VSM-based analysis allows one to integrate concepts and instruments which emanate from other approaches. For example, the analysis of roles such as "client", "problem solver" and "problem owner", as used in SSM (Checkland and Winter, 2006), can enhance the understanding of an intervention process.

For the sake of illustrating this point, we refer to the example of the RITTS project already mentioned. In that instance, ensuring the viability of the project was crucial. Conclusively, a solid diagnosis was required in the first place. The VSM was used for that purpose, and it brought to the fore extremely valuable diagnostic points (Figure 5).

In brief, the VSM-based scrutiny revealed the following diagnostic points. In contrast to the normative management (System 5)—embodied by a steering committee-which had a clear policy and a firm basis of shared values, the strategic intelligence (System 4) had an ephemeral character. It only existed temporarily in the form of workshops dedicated to strategic analyses. The operative management had a strong coordination function to dampen oscillations-a local agency for innovation and technology transfer (System 2)—but 
Fig. 5. VSM-model at the level of the organizational context (example)

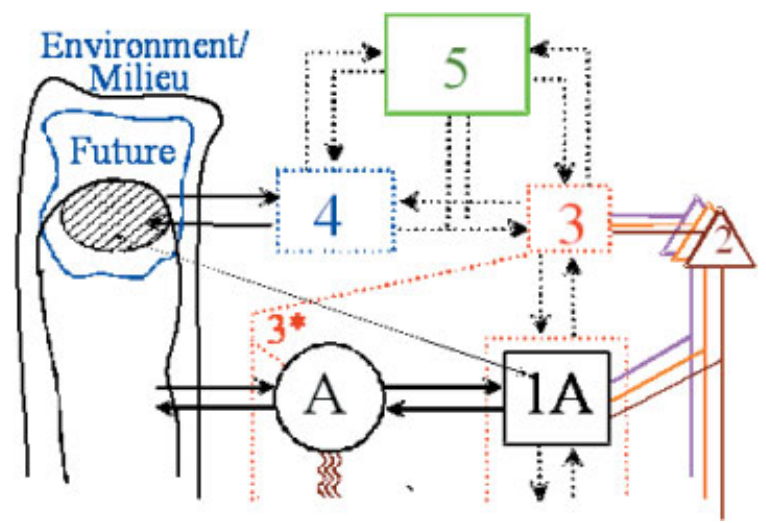

the overall (operative) management function (System 3) was still weak. Also, the links between the overall control function and the primary units (basic units with regulatory capacity, i.e., System 1), and the monitoring/auditing function (System $3^{*}$ ) were ill defined. As far as the basic operational units were concerned, there was some ambiguity as to the criteria for defining them. For a start, it made good sense to use the geographical criterion implying a structure among political districts.

The recommendations derived from that diagnosis were straightforward. Above all, the management structure of the RITTS project needed to be strengthened substantially. Both Systems 3 and 4, which were recognizable only in a rudimentary manner, needed to be enacted properly. The respective agents had to be identified, and provided with clear tasks and responsibilities, in order to be effective.

In sum, the VSM enabled a clear diagnosis which went beyond what can usually be achieved with more traditional approaches. The example also makes visually apparent that the VSM shows other strengths than SD, and that these are highly complementary to the dynamic analyses provided by SD modeling. The distinctive contribution of the VSM in this case was a set of diagnostic points about the organizational context of the RITTS- the ones just enumerated. At the same time, the VSM diagnosis clearly indicated that use of the SD model as a management support tool within the organization was highly unlikely as long as System 4 would not be strengthened.

Let us suppose, alternatively, that this case would have been viewed exclusively through the lens of the VSM. The VSM-based organizational diagnosis in itself would have secured useful results, triggering insights into how the management of the RITTS project should be structured. However, it would not have provided or fostered an understanding of how the regional innovation and transfer system functioned and how its takeoff should be brought about. For that purpose a dynamic simulation model was necessary. This shows not only 
that but also how the VSM needs SD as a complementary methodology. The complementarity is not a one-way street, on which only SD has need of the VSM.

\section{SD and the VSM: strategy making in the 3-4 homeostat}

In principle, SD models would be applicable in any of the management subsystems specified by the VSM. Given the limited size of this paper, we shall concentrate on one crucial example: the interaction of Systems 3 and 4. That interaction is the "place" of strategy making. We choose this example because strategy is one of the most fertile domains for SD applications and at the same time is decisive for the sustained viability of an organization. Therefore, this instance illuminates the complementarity of SD and the VSM better than other examples might do.

Above, we have used the term "outside and then" for System 4 in the VSM. Similarly a description of System 3, in connection with Systems 2 and 1, is "management of the inside and now". While the first is about the development of the organization as a whole in the middle and the long term, the latter is about efficient response to instant requirements on a continual basis. Without a doubt, these are two different types of issues. Both domains have their distinctive logic and specific goals, and they need different "languages" to deal with them.

Systems 3 and 4 interact in such a way as to balance their respective varieties (i.e., their repertoires of behavior). Each one generates high variety, much of which is absorbed in this interaction (Beer, 1979, p. 261). In other words, each of them shows behaviors, presents arguments, etc. System 3 is marked by imperatives of the short term and the inside of the organization, and System 4 by those imperatives which derive from a long-term and environment-oriented view. Their interplay should entail mutual alignment. This becomes difficult if some of the variety remains unattended to ("residual variety"; Espejo, 1989). It is the task of the logically higher organizational system-System 5, in this case-to regulate for equilibrium (e.g., moderating the process). In practice, this kind of interaction takes place, e.g., in workshops which bring together the exponents of corporate management in charge of, say, production and marketing (for System 3) with those of research and corporate planning (for System 4). The former are rather short-term, the latter long-term oriented. If they do not reach mutual alignment, the CEO (for System 5) will have to intervene.

If Systems 3 and 4 do not interact properly, the viability of the organization will be in danger, due either to a lack of contact with actual affairs (dominance of System 4) or to an excessive prevalence of day-to-day interests (dominance of System 3). Hence, the organization will sooner or later suffer from schizophrenia. ${ }^{28}$ More precisely, both the goals emanating from Systems 3 and 4 will be inflicted upon the operational units (System 1), at the same time and independently of each other. This implies that the imperatives of the short and the long term will be imposed concurrently. The likely contradictions between 
the two positions will propagate instead of being submitted to checks and balances, at an earlier stage, within the meta-system 3-4-5 (Schwaninger, 2006b). Hence, a proper interaction of Systems 3 and 4 (moderated by System 5 ) is a must; it should be a genuine dialogue about the organization's future and how to bring it about.

But if both systems-3 and 4-have different languages, how can they engage in a fertile dialogue? The systemic solution is the creation of an interface which transduces the categories of one domain into the language of the other and vice versa. This is precisely what a good SD model can provide. In such a model, different kinds of variables, e.g., economic, technological, social and ecological, can be integrated, so that the distinct perspectives of the short and the long term can be connected to each other.

The interaction between Systems 3 and 4 can be about different things. Three major types of issues are pervasive:

1. Translating strategy options into operative categories and assessing their consequences.

2. Examining the implications of operations for the development of the firm, in terms of possibilities and restrictions.

3. Trade-offs between the short and the longer term; priorities for and consequences of decisions.

For all three purposes, simulation models are, in principle, a necessary tool. It is precisely at the interface of strategy and operations, i.e., at the intersection of the Systems 3-4-5 process and the Systems 1-2-3 interaction, that SD can make its strongest contribution. There it fulfils the crucial task of showing the implications of strategic decisions for the operative domain. Here, quantification is crucial, e.g., for deriving investment needs, liquidity bottlenecks, etc. This does not preclude the fact that SD also remains very important at both ends, for System 4 (concerning long-term scenarios about market, technologies, etc.) and System 3 (relative to the allocation of resources, the balance, the sequencing and scheduling of investments, etc.).

At both strategic and operative levels, the exploration of scenarios based on dynamic models can provide insights and clues, which are an important complement to exclusively qualitative deliberations. Similarly and even more importantly, trade-offs merely pondered in qualitative terms can hardly be dealt with competently, whereas making them tangible in quantitative terms is often very helpful. Finally, strategy designs, which transcend the status quo, can be made more discussible, if the discourse is accompanied and supported by an SD modeling exercise. Changes in the mental models will be made explicit and will lead to changes in the SD models, which immediately feed back results. This way, the intuition and judgment of decision makers can be honed in interaction with the model. Such a process should even breed rich insights, if this model-based discourse is carried out over a longer period. 
Recurrently, conjectures and decisions will be followed by proofs and refutations, and a learning experience will ensue. Ultimately, this should lead to better strategies.

The VSM in this connection helps make sense of the decisions taken by translating them into the distinct languages of the inside-and-now (System 3) and the outside-and-then (System 4). In addition, it enables management to figure out if the organization is prepared for an effective realization of decisions in the current or future organizational context.

The variety absorption process in the meta-system 3-4-5 of an organization is, in principle, the same phenomenon as the process of mutual alignment in GMB, as described in the section about SD modeling. Essentially, the different perspectives mentioned there with regard to distinct disciplines can also be ascribed to the representatives of Systems 3, 4, 5, who have to arrive at a shared understanding as well. ${ }^{29}$ This invariant feature of modeling, across both approaches SD and VSM, is an indication of the need for integrating the two.

\section{When and how combine SD and VSM?}

We are dealing with two questions here. The first pertains to contingency: when-under what circumstances-should SD and the VSM be combined?

If we claim that SD and VSM should be integrated, we are proposing a principle. We are not suggesting, however, that such an integration must actually take place in each and every case of an SD project or VSM application. A combined use of the two is not an unconditional necessity. Such a combination is indicated, i.e., it delivers value, only under certain conditions. We limit ourselves to the establishment of two criteria.

One criterion is the interaction between the issue under study ("content") and the organization ("context"). A second criterion is the functionality of the current organizational structure. If the organizational structure is affected strongly by the issue at hand or vice versa, or if it shows significant disfunctionality, then the model of the issue (SD) should be complemented by an organizational diagnosis (VSM). In the absence of such a strong interrelationship and of disfunctionality, an additional organizational modeling is, in principle, not required, even though such an extra effort might have its benefits.

Another criterion is the nature of the organizational context in which the modeling project operates. If that context is simple (e.g., small organization, unitary decision power), then a special VSM diagnosis is not necessary. If that organizational context per contra is complex, then a use of the VSM is indicated. This applies to knotty organizations with many stakeholders, or to cases in which many actors are affected by the modeling exercise, or in which multiple opinions among those actors exist. A further indication of such complexity could be a strong interaction between the model and the organization, for example, if the model has a strong (potential) impact on either the organization or some of its parts. 
The second question to be dealt with here concerns the how of a complementary approach. This question touches on the practical dimension of the complementarity - that is, should one start with a VSM diagnosis or with an SD analysis?

Specifically, does one need to start with a VSM diagnosis followed by an organizational redesign with respect to structures which are problematic in terms of viability? Or should one start with an SD project and then continue with a viability analysis?

Our stance rests on the equifinality principle (von Bertalanffy, 1968): one and the same goal can be attained via different pathways. This should not be interpreted as a plea for arbitrariness. There are indeed contingencies to be taken into account.

The first point is a profoundly systemic one: one should not try to solve a problem where it arises. In the ideal case, if a client calls for the solution of a specific problem, the consultant should analyze the bigger picture together with him or her. For that purpose, if the issue at hand is complex, a qualitative SD approach (mapping with the help of causal loop diagrams-CLDs) is often the most powerful way of gaining an overview. From there it will become apparent if the next steps should be either further SD work or VSM analysis. The respective activities often can be tackled in parallel, but one must avoid overwhelming the client.

A sensible alternative is to begin with one of the two approaches, if the priorities are clear at the outset or if there are restrictions such as acute resource scarcity. If emphasis falls on the SD track, then a VSM analysis will often be useful as a complement, to enable a deeper understanding and more effective action at the implementation stage. If the VSM track comes to the fore, CLDs or small SD models can fulfill the important supplementary role of increasing the effectiveness of the intervention.

By the way, group model building will be indicated theoretically in any case, be it with SD, VSM or complementary methodology. So also will important qualitative analyses and assessments, such as the extended cultural analysis as proposed by Lane and Oliva (1998), much of which can be captured in CLDs.

We refrain from giving a definite prescriptive chart on how to proceed, and instead refer to a more general heuristic scheme presented elsewhere as a help for the complementary use of SD and the VSM (Schwaninger, 2004).

To summarize, we are proposing that a combination of the two methodologies is not necessary in all cases. We do, however, claim that a potential combination should be considered when one uses either of these methodologies in dealing with a complex issue. This claim implies that the respective specialists would have to include both methodologies in their repertoire.

Finally, we will briefly elaborate on how the methodological integration can be facilitated by software support. 


\section{Software tools}

The advantages of a combined use of the VSM and SD were seen early on. When Beer presented his VSM in Brain of the Firm, he already mentioned the convenience of using Forrester's SD and the corresponding software programs together with his model (Beer, 1981, p. 197).

Each one-VSM-based diagnosis and design on the one hand and SD on the other-is a powerful methodology in its own right. We have pleaded for their combination because of the potential synergies. Yet there is no sensible way of combining them in an algorithmic or mechanical way. A human integrator is needed. What can be done, however, is to support those interested in combining the two methodologies by supplying software tools that facilitate their handling, especially the model logistics involved.

While the SD community has come up with powerful software packages to support modeling and validation, such assistance for VSM-based modeling has been practically nonexistent. This is about to change. Under the leadership of one of the authors (J.P.R.) a software has been developed, VSMod, to facilitate the application of the VSM and its combination with SD.

VSMod facilitates the design of models for organizations in a manner familiar to SD modelers. The modeling technique is screen-driven, with iconic representations. The underlying recursive design facilitates the creation of VSMbased structures, according to multiple recursion criteria, and the uploading of information at any level and in any component of the model. VSM-based modeling with VSMod is particularly helpful in the cases where multiple units and recursion levels must be represented. For a detailed description of the functionality, see Pérez-Ríos (2003, 2006).

Figure 6 shows a screenshot of the software. It refers to the case of a Brazilian media group, where one of the authors (M.S.) carried out a VSM-based analysis of the corporate structure. On the left of the diagram is the representation of the corporation's organization at Recursion Level One, with three divisions (Publications, etc.). To the right, the embedding of the organizational units of different recursion levels ("father system”, "child system”) are visible. Moving along either the different levels or across the parallel systems is one of the fundamental functions provided by the software. A multilevel model can be constructed, starting from anywhere, at the top, bottom, or intermediate levels.

VSMod is an effective tool for integrating VSM and SD because it provides researchers or practitioners with a facility for inserting the SD model into the VSM environment while working inside the VSM model. They do not have to leave the VSM environment in order to make the integration. Moreover, they can proceed this way at any level of recursion. This means that they can use as many SD models as needed within a VSM study.

One of the contributions of VSMod to an integration of SD and VSM is the provision of a repository in which SD models can be stored and used. For example, in the case of a multi-recursion enterprise, the representatives of 


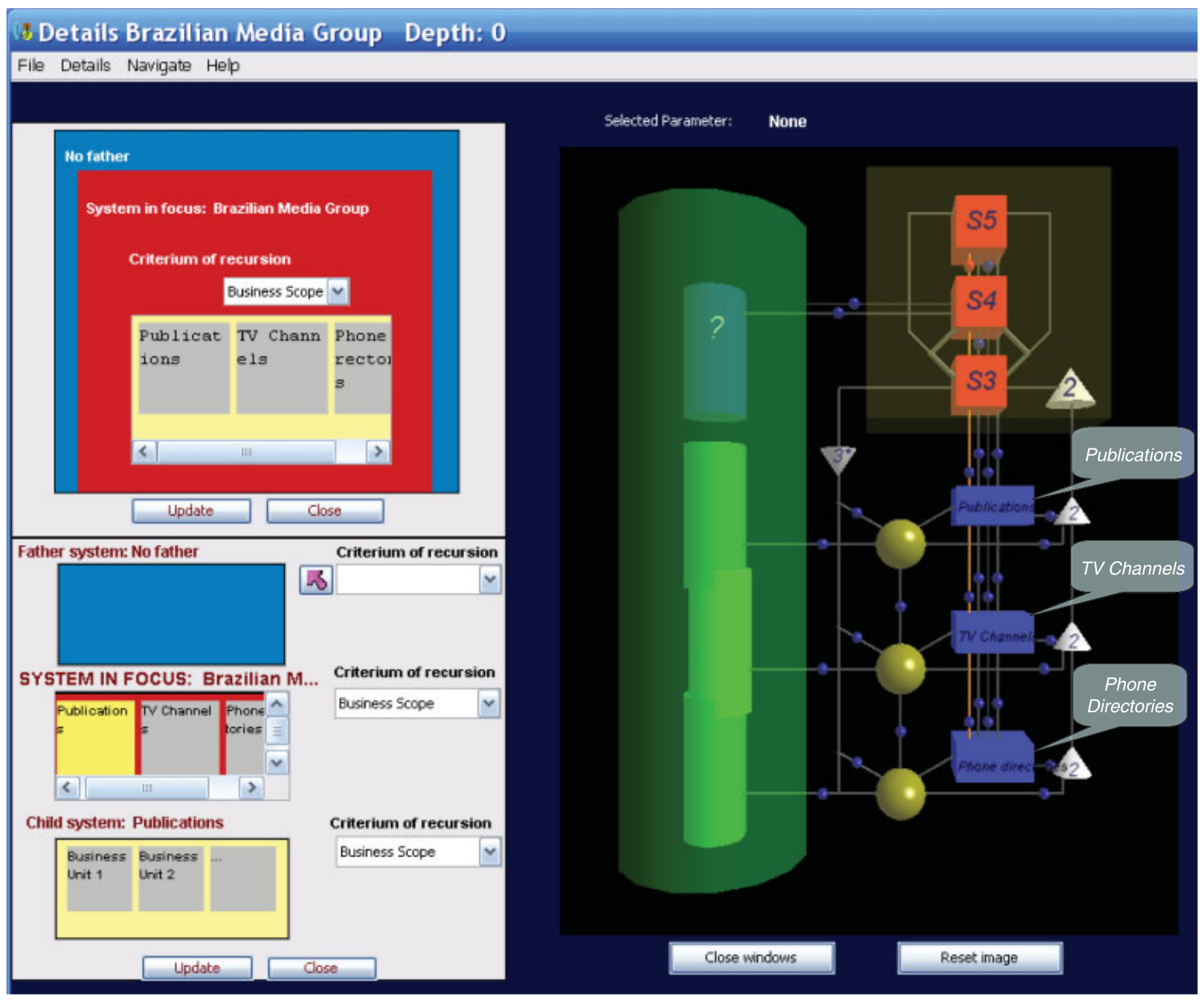

Fig. 6. Screenshot of VSMod ${ }^{\circledR}$ : example of a Brazilian media group

Systems 4 at the different levels can deposit their respective SD models on the software platform and call upon them whenever necessary. Different models can be distributed anywhere in the VSM model system, so that actors in different places can construct their models locally, while making them available in the repository for shared use, e.g., in strategy workshops or for employment by System 4 exponents. In the multi-user version, users in different locations of the organization have access to one and the same model system. This way, both content and context related models (as specified in Figure 2) can be readily accessible anywhere in the organization. 
As far as the alignment and the integration of different SD models are concerned, these are not meant to be achieved mechanically. They call for a dialogue in the first place. Secondly, couplings of SD models from different locations within the VSM can only be achieved if their architecture is modular. More experience on that topic will have to be gathered in the future.

So far, the VSMod software is being used and tested in projects of applied research, by a substantial number of corporations and institutions, where the combination of VSM and SD is being explored. These experiments are likely to lead to improvements at the software end. The software can be made available for testing purposes by one of the authors (J.P.R.).

\section{Conclusions}

We have outlined the similarities and differences of both system dynamics (SD) and organizational cybernetics (OC), the latter with particular attention to the viable system model (VSM). Drawing on analysis of the strengths and limitations of both methodologies, we have argued that the two approaches could result in additional benefits if combined. We even claim that the synergy between SD and the VSM is (almost) a necessary one, if a more complete approach to deal with complex organizational issues is needed.

SD has been identified as a very powerful device for dealing with complexities at the level of the content of organizational activities, by modeling and simulating them dynamically. For the modeling and design of the organizational context so as to enable the viability of the system under study, we have identified the VSM as an unmatched conceptual and methodological device which can open new possibilities to the discipline of SD. We have also introduced a new software package-VSMod-which supports the combination of SD and VSM models on the same platform.

We have emphasized the complementarity of SD and VSM, which can strengthen each other. Two addenda are necessary. First of all, the two methodologies cannot be fused in an algorithmic way. However, they can be put to a combined use and become a powerful pair. ${ }^{30}$ Secondly, we do not suggest that SD modeling and the VSM are the only methodologies available to deal with complex organizational issues. There are certainly other methods and methodologies that can be used and combined.

We have identified SD and OC, namely the VSM, as candidates with a marked synergetic potential, as far as applications to organizations and society are concerned, for several reasons:

1. Both of them are rooted in the systems approach, which represents a new stage in the evolution of science ${ }^{31}$ - a stage of adaptation to new levels of complexity.

2. Both are highly generic and therefore applicable to a great variety of situations.

3. Their objectives are complementary and in harmony. 
4. Their methodologies are individually incomplete, in nuce mutually exclusive, but collectively rather comprehensive.

5. They are connectable in functional and virtuous ways.

Therefore, in our view, the potential synergy between system dynamics and organizational cybernetics is a powerful one. In order to reap the benefits of that synergy, however, it is practically necessary to put it into action.

\section{Notes}

1. We refrain from using the term "paradigms", which we consider to apply to the fundamental beliefs and assumptions shared by a scientific community (Kuhn, 1996).

2. The terms organizational cybernetics and managerial cybernetics will be treated as synonyms here, as subtle differences of the two terms-mostly for historical reasons-are not relevant in the context of this paper.

3. When referring to SD methodology we include all phases of the SD-based approaches to problem solving, e.g., as proposed by Richardson and Pugh (1981, p. 16): problem identification and definition, system conceptualization, model formulation, analysis of model behavior, model evaluation (including validation), policy analysis, model use or implementation. Also, related methodological issues such as group model building and the pertinent heuristics (Vennix, 1996) are, in our view, part of SD methodology.

4. For an alternative although similar methodological design see Checkland and Winter (2006), who suggest analyzing at two levels, for "content of the problematical situation" and "the process of the intervention itself" (p. 1435). In the field of strategy, the distinction of content and processrelated research is commonly used (Lechner, 2006, p. 7).

5. To date, the scientific attempts to falsify the model (particularly Frost, 2005; Crisan Tran, 2006) have been unsuccessful.

6. See, for example, a special edition of the journal Systems Research and Behavioral Science, published under the title "System dynamics in organisational consultation: modelling for intervening in organizations”, Vol. 23, No. 4, July-August 2006. See also Pettigrew et al. (2001) and Poole et al. (2000).

7. For an overview, see Richardson (1991); Jackson (2000, p. 142ff.).

8. SD has been applied to the most diverse subject areas, e.g., global modeling, environmental issues, social and economic policy, corporate and public management, regional planning, medicine, psychology and education in mathematics, physics and biology.

9. Of ISEE Systems (formerly High Performance Systems Inc.), Powersim A/ S, Ventana Systems Ltd., and Strategy Dynamics Ltd, respectively.

10. Lane (2006, p. 484) has termed SD as "one of the most widely used systems approaches in the world". 
11. The notion of structure as a system of relationships is based on a cybernetic concept of organization, which emphasizes the importance of relations, assigning the system's elements to a secondary plane (Beer, 1966, p. 425f.; Drucker, 1979, p. 439ff.).

12. "Variety" is a technical term for "complexity", which denotes a (high) number of potential states or behaviors of a system (based on Ashby, 1956).

13. Ashby's Law of Requisite Variety says: "Only variety can destroy variety”, which implies that the varieties of two interacting systems must be in balance, if stability is to be achieved (Ashby, 1956).

14. The term "variety engineering" has been used in this context (Beer, 1979).

15. For an overview, see Gomez (1981), Jackson (2000, p. 172ff.) and Malik (2002).

16. For the following, see Beer $(1979,1981,1985)$.

17. This is a slightly simplified version (Beer, 1985).

18. We are abstracting from the distinction between structure and organization as it is made in the biological literature (Varela, 1979).

19. The conceptual basis of this postulate is the Conant-Ashby theorem (Conant and Ashby, 1981).

20. Schwaninger $(1997,2004)$ has elaborated on these complementarities.

21. Group model building, as understood here, is a methodology to facilitate team learning with the help of SD (Vennix, 1996). The methodology consists in a set of methods and instruments as well as heuristic principles. These are meant to facilitate the elicitation of knowledge, the creation of a shared understanding of a problem in a team, and the joint building of models.

22. Theory of planned behavior; see, for instance, Ajzen (1991).

23. More details of that project have been documented in Pérez Ríos and Schwaninger (1996) and Schwaninger (1997).

24. Organizational blindness is a strong indicator of organizational decline (Weitzel and Jonsson, 1989).

25. This holds for both biological systems (Worm et al., 2006) and social systems (Olaya, 2007).

26. The underlying problem here is "not seeing that one is not seeing" (von Foerster, 1984, p. 4).

27. The attribute "social" here refers to both structural and cultural aspects. Although the VSM is primarily a structural model it nonetheless embraces the cultural dimension of analysis as well (see description of System 5 above).

28. From Greek schizein, to split, and phren, mind, spirit.

29. In the end, a meta-system needs a model which encompasses all three perspectives, and the fulcrum for such an overall model is System 4, as already explained.

30. A heuristic called "integrative systems methodology" has been proposed elsewhere, as a helpful device for the combination of SD and the VSM 
(Schwaninger, 1997, 2004). It is a framework that shows how the synthesis of methodologies advocated here can be achieved, although at a highly abstract level. Therefore this paper provides more concrete methodological building blocks to make that framework operational and achieve progress towards the postulated synergy.

31. This claim has been made by Anatol Rapoport, one of the founding fathers of systems theory (personal communication, 1998).

\section{References}

Ajzen I. 1991. The theory of planned behaviour. Organisational Behaviour and Human Decision Processes 50: 179-211.

Andersen DF, Richardson GP. 1997. Scripts for group model building. System Dynamics Review 13(2): 107-129.

Ashby WR. 1956. An Introduction to Cybernetics. Chapman \& Hall: London.

Beer S. 1966. Decision and Control. Wiley: Chichester.

—. 1979. The Heart of Enterprise. Wiley: Chichester.

— 1981. The Brain of the Firm. Wiley: Chichester.

—. 1985. Diagnosing the System for Organizations. Wiley: Chichester.

- 1989. The viable system model: its provenance, development, methodology and pathology. In The Viable System Model: Interpretations and Applications of Stafford Beer's VSM, Espejo R, Harnden R (eds). Wiley: Chichester; 11-37.

Checkland PB. 1981. Systems Thinking, Systems Practice. Wiley: Chichester.

Checkland P, Winter M. 2006. Process and content: two ways of using SSM. Journal of the Operational Research Society 57: 1435-1441.

Conant RC, Ashby WR. 1981. Every good regulator of a system must be a model of that system. In Mechanisms of Intelligence: Ashby's Writings on Cybernetics, Conant RC (ed.). Intersystems Publications: Seaside, CA; 205-214.

Crisan Tran CI. 2006. Beers Viable System Model und die Lebensfähigkeit von Jungunternehmen: Eine empirische Untersuchung. PhD dissertation No. 3201, University of St Gallen, Switzerland.

Drucker PF. 1979. Management. Pan: London.

Eberlein RL, Peterson DW. 1994. Understanding models with Vensim. In Modeling for Learning Organizations, Morecroft JDW, Sterman JD (eds). Productivity Press: Portland, OR; 339-348.

Espejo R. 1989. The VSM revisited. In The Viable System Model: Interpretations and Applications of Stafford Beer's VSM, Espejo R, Harnden R (eds). Wiley: Chichester; 77-100.

- 1993. Management of complexity in problem solving. In Organisational Fitness: Corporate Effectiveness through Management Cybernetics, Espejo R, Schwaninger M (eds). Campus: Frankfurt; 67-92.

Forrester JW. 1961. Industrial Dynamics. MIT Press: Cambridge, MA. ${ }^{+}$

— 1969. Principles of Systems. Wright-Allan Press: Cambridge, MA. ${ }^{\dagger}$

${ }^{\dagger}$ Now available from Pegasus Communications, Waltham, MA. 
1971. World Dynamics. Productivity Press: Portland, OR. ${ }^{\dagger}$

. 1993. System dynamics as an organizing framework for pre-college education. System Dynamics Review 9(2): 183-194.

- 1997. System dynamics and K-12 teachers. Lecture, University of Virginia School of Education, Massachusetts Institute of Technology, System Dynamics Group, Paper D-4665-4.

Frost B. 2005. Lebensfähigkeit von Communities of Practice im organisationalen Kontext. $\mathrm{PhD}$ Dissertation, University of St Gallen, Switzerland.

Gomez P. 1981. Modelle und Methoden des systemorientierten Managements. Haupt: Bern.

Jackson MC. 1988. An appreciation of Stafford Beer's "Viable System" viewpoint on managerial practice. Journal of Management Studies 25(6): 557-573.

—. 2000. Systems Approaches to Management. Kluwer: New York.

Janis IL. 1972. Victims of Groupthink: A Psychological Study of Foreign Policy Decisions and Fiascos. Houghton Mifflin: Boston, MA.

Kuhn TS. 1996. The Structure of Scientific Revolutions (3rd edn). University of Chicago Press: Chicago.

Lane DC. 2006. IFORS' operational research hall of fame: Jay Wright Forrester. International Transactions in Operational Research 13: 483-492.

Lane DC, Oliva R. 1998. The greater whole: towards a synthesis of system dynamics and soft systems methodology. European Journal of Operational Research 107(1): 214-235.

La Roche U, Simon M. 2000. Geschäftsprozesse simulieren: flexibel und zielorientiert führen mit Fliessmodellen. Orell Füssli: Zürich.

Lechner C. 2006. A Primer to Strategy Research. Cuvillier: Göttingen.

Malik F. 2002. Strategie des Managements komplexer Systeme (7th edn). Haupt: Bern.

Meadows DH. 1980. The unavoidable à priori. In Elements of the System Dynamics Method, Randers, J (ed.). MIT Press: Cambridge, MA; 23-57. ${ }^{+}$

Meadows DH, Richardson J, Bruckmann G. 1982. Groping in the Dark: The First Decade of Global Modelling. Wiley: Chichester.

Mingers J, Gill A. 1997. Multimethodology: The Theory and Practice of Combining Management Science Methodologies. Wiley: Chichester.

Odum HT, Odum EC. 2000. Modeling for all Scales: An Introduction to System Simulation. Academic Press: San Diego, CA.

Olaya C. 2009. Evolutionary governance. PhD dissertation, University of St Gallen, Switzerland (forthcoming).

Oxford English Dictionary. 1996. The Compact Oxford English Dictionary (2nd edn). Clarendon Press: Oxford.

Pérez Ríos J. 2003. VSMod: a software tool for the application of the viable system model. In Proceedings, 47th Annual Conference of the International Society for the Systems Sciences (ISSS), Heraklion, Crete, Greece.

Pérez Ríos J. 2006. Communication and information technologies to enable viable organizations. Kybernetes 35(7/8): 1109-1125.

Pérez Ríos J, Schwaninger M. 1996. Integrative systems modeling: leveraging complementarities of qualitative and quantitative methodologies. In Proceedings, 1996

\footnotetext{
${ }^{\dagger}$ Now available from Pegasus Communications, Waltham, MA.
} 
International System Dynamics Conference, Vol. 2, Richardson GP, Sterman JD (eds). Cambridge, MA, 21-25 July 1996; 431-437.

Pettigrew AM. 1985. The Awakening Giant: Continuity and Change in Imperial Chemical Industries. Blackwell: Oxford.

Pettigrew A, Woodman RW, Cameron K. 2001. Studying organizational change and development: challenges for future research. Academy of Management Journal 44(4): 697-713.

Poole MS, Van de Ven AH, Dooley KJ, Homes ME. 2000. Organizational Change and Innovation Processes. Oxford University Press: New York.

Richardson GP. 1991. Feedback Thought in Social Science and Systems Theory. University of Pennsylvania Press: Philadelphia.

Richardson GP, Pugh AL. 1981. Introduction to System Dynamics Modeling with DYNAMO. MIT Press: Cambridge, MA. ${ }^{+}$

Richmond B. 1997. The "thinking" in systems thinking: how can we make it easier to master? Systems Thinker 8(2): 1-5.

Schwaninger M. 1997. Integrative systems methodology: heuristic for requisite variety. International Transactions in Operational Research 4(4): 109-123.

- 2004. Methodologies in conflict: achieving synergies between system dynamics and organizational cybernetics. Systems Research and Behavioural Science 21(4): 411-431.

- 2006a. Theories of viability. a comparison. Systems Research and Behavioural Science 23: 337-347.

—. 2006b. Design for viable organizations: the diagnostic power of the viable system model. Kybernetes 35(7/8): 955-966.

Schwaninger M, Pérez Ríos J, Ambroz K. 2004. System dynamics and cybernetics: a necessary synergy. In Proceedings, International System Dynamics Conference, Oxford, Kennedy M et al. (eds). System Dynamics Society: Albany, NY. (CD-ROM).

Sterman JD. 1994. Learning in and about complex systems. System Dynamics Review 10: $291-330$.

- 2000. Business Dynamics: Systems Thinking and Modeling for a Complex World. Irwin/McGraw-Hill: Boston, MA.

Varela F. 1979. Principles of Biological Autonomy. North-Holland/Elsevier: New York. Vennix JAM. 1996. Group Model Building: Facilitating Team Learning Using System Dynamics. Wiley: Chichester.

Von Bertalanffy L. 1968. General System Theory: Foundations, Development, Applications. Braziller: New York.

Von Foerster H. 1984. Principles of self-organization: in a socio-managerial context. In Self-Organization and Management of Social Systems. Springer: Berlin; 2-24.

Walker J. 2001. The viable systems model: a guide for co-operatives and federations. www.greybox.uklinux.net [28 July 2006].

Weitzel W, Jonsson E. 1989. Decline in organization: a literature integration and extension. Administrative Science Quarterly 34: 91-109.

Worm B, Barbier EB, Beaumont N, Duffy JE, Folke C, Halpern BS, Jackson JBC, Lotze HK, Micheli F, Palumbi SR, Sala E, Selkoe KA, Stachowicz JJ, Watson R. 2006. Impacts of biodiversity loss on ocean ecosystem stability. Science 314(3): 787-789.

${ }^{\dagger}$ Now available from Pegasus Communications, Waltham, MA. 